\title{
Satisfacción con la imagen corporal y calidad de vida en mujeres con reconstrucción mamaria por cáncer: una revisión sistemática
}

\author{
Body image satisfaction and quality of life in women \\ with mammary reconstruction after cancer: $A$ systematic review
}

\author{
Martha Juliana Villegas Moreno y Constanza Londoño
}

Citación: Villegas M., M.J. y Londoño, C. (2019). Satisfacción con la imagen corporal y calidad de vida en mujeres con reconstrucción mamaria por cáncer: una revisión sistemática. Psicología y Salud, 29(2), 259-268.

\section{RESUMEN}

\begin{abstract}
El cáncer de mama, enfermedad que causa el mayor número de muertes en las mujeres, acarrea devastadoras consecuencias para su calidad de vida, más aún cuando se someten a una mastectomía, tras de la cual se les propone generalmente la reconstrucción mamaria, bien sea con implantes, expansores o colgajos. El propósito de esta revisión sistemática fue identificar el efecto que tiene dicha reconstrucción en la satisfacción con la imagen corporal y la calidad de vida de estas mujeres, revisión que se llevó a cabo utilizando diferentes bases de datos mediante la combinación de palabras clave en los idiomas español e inglés. Como resultado, se encontraron 290 artículos que cumplían con los criterios de selección. Al aplicar la prueba DIAD, se seleccionaron solamente cinco artículos que cumplían con los parámetros de calidad para la investigación en intervenciones clínicas. A partir de tales estudios, se concluye en general que la reconstrucción mamaria tipo TRAM genera un mayor nivel de satisfacción con la imagen corporal y una mejoría en la calidad de vida; sin embargo, el ejercicio de la sexualidad continúa afectado en tales mujeres. Por último, se señala que el hecho de involucrarse de manera activa en el proceso y en la toma decisional de las pacientes tiene efectos positivos en su calidad de vida.
\end{abstract}

Palabras clave: Cáncer de mama; Mastectomía; Imagen corporal; Calidad de vida; Reconstrucción mamaria; Revisión sistemática.

\begin{abstract}
Breast cancer causes the greatest number of deaths for women and produces devastating consequences for their quality of life, especially when a mastectomy is performed. In this circumstance breast reconstruction is usually proposed, either through implants and expanders or with flaps. The purpose of the present systematic review was to identify the effect of breast reconstruction on body satisfaction, body image and quality of life. A systematic search was conducted in diverse databases, combining keywords in both Spanish and English. An initial total of 290 articles met the selection criteria. When the DIAD Test, was applied, only five articles met the quality parameters for research in clinical interventions and were selected. As a general conclusion, TRAM type breast reconstruction generates a higher level of satisfaction with body image and improves quality of life; however, the exercise of sexuality continues being affected in these women. Finally, it can be pointed out that being actively involved in the process and in decision-making process positively contributes to better patients' quality of life.
\end{abstract}

Key words: Breast cancer; Mastectomy; Body image; Quality of life; Breast reconstruction; Systematic review.

\footnotetext{
${ }^{1}$ Programa de Psicología, Universidad Católica de Pereira, Carrera 21, Núm. 49-95, Av. de las Américas, Pereira (Colombia), tel. (57)63-12-40-00, correo electrónico: mjvillegas16@ucatolica.edu.co. Artículo recibido el 2 de agosto y aceptado el 23 de noviembre de 2018.

${ }^{2}$ Doctorado en Psicología, Facultad de Psicología, Universidad Católica de Colombia, Av. Caracas 46-72, Bogotá (Colombia), tel, (57)13-27-73-00, correo electrónico: clondono@ucatolica.edu.co.
} 
$\mathrm{E}$ $\mathrm{s}$ bien sabido que el cáncer es la principal causa de muerte en el mundo. Según la Organización Mundial de la Salud (OMS), en el año 2015 ocasionó 8.8 millones de defunciones, siendo el cáncer de mama la más importante causa de muerte por cáncer en las mujeres (OMS, 2017). La Organización Panamericana de la Salud, la Sociedad Médica Oncológica de Latinoamérica y la OMS estiman que en el año 2030 habrá más de 596 mil nuevos casos y más de 142 mil muertes por esta enfermedad en América Latina y el Caribe (cf. Agencia Internacional de Investigación en Cáncer, 2012).

Hay diferentes tipos de tratamientos para el cáncer de mama, entre ellos los quirúrgicos (mastectomía) (Álvarez et al., 2015; Marré, Gantz, Villalón y Roco, 2016; Román, 2007; Yustos, Angulo y Soto, 2017), que van de radicales a conservadores (Cruz y Morales, 2014). Entre los primeros, la mastectomía consiste en la extirpación de la mama, cuya secuela física inmediata son los diferentes conflictos con la autoimagen en virtud de que dicha operación trae consigo una importante deformidad física del cuerpo, y visualmente supone serias consecuencias emocionales (como depresión y ansiedad), cognitivas y comportamentales (Bindu y Baboo, 2017; Martínez, Lozano, Rodríguez, Galindo y Alvarado, 2014; Segura, García y Gutiérrez, 2014).

Los senos son concebidos culturalmente como un símbolo de la mujer, la maternidad, el atractivo físico, la feminidad, el erotismo, la sensualidad, la sexualidad y la capacidad reproductora, entre otros. Es por ello que las consecuencias de perder uno o ambos senos repercuten de manera drástica en la imagen corporal y en la calidad de vida de la mujer (Cucarella, 2013; Gallegos, Pérez y Bustamante, 2017; Morales, Torres y Vega, 2016; Tumas, Díaz y Carbonetti, 2013; Villamil et al., 2012).

La pérdida ocasionada por la intervención quirúrgica provoca en efecto cambios drásticos en la apariencia corporal y deja importantes cicatrices, deformidades y linfedemas (hinchazones), lo que afecta la capacidad de lactar, la sensibilidad, la movilidad de los brazos o su entumecimiento, entre otros efectos propios de la cirugía (Bruant-Rodier y Bodin, 2014; Fernández, 2004; Mejía, 2012).
No obstante, las mujeres pueden someterse a una nueva cirugía para reconstruir la forma del seno y con ello recuperar una apariencia corporal positiva (Instituto Nacional del Cáncer, 2016). Hay dos técnicas principales para reconstruir la mama: la reconstrucción con implante y la reconstrucción autóloga o con colgajo. Respecto a esta última, hay diferentes tipos de procedimiento: implante que se hace de forma inmediata, expansión seguida de implante que puede ser inmediato o retrasado, colgajo de músculo recto transverso del abdomen (TRAM), colgajo del músculo recto transverso del abdomen libre sin afectación de músculo (DIEP), colgajo de arteria epigástrica superficial inferior (SIEA), perforante de la arteria glútea (GAP por sus siglas en inglés) o perforante de la arteria femoral profunda (PAP, también por sus siglas en inglés). Estos últimos procedimientos pueden realizarse inmediatamente o un tiempo después de ocurrida la cirugía (Breastcancer, 2016).

A pesar de ser un procedimiento simple, la reconstrucción mamaria con implantes tiene resultados estéticos o naturales más pobres, así como un elevado índice de complicaciones, como seromas o necrosis cutáneas, aunque autores como Negenborn et al. (2018) hallan que la intervención por medio de esta técnica genera una mayor satisfacción para la paciente que el uso de matrices dérmicas celulares. En la reconstrucción autóloga, esto es, con colgajos o tejidos propios, se presentan necrosis del colgajo, grasa, hernias y eventraciones, por lo que hay algunas contraindicaciones para su realización, como tener antecedentes de TRAM o abdominoplastía, de vaciamiento axilar con radioterapia asociada, toracotomías o índice de masa muscular mayor a 28 (Sepúlveda, 2016).

Es evidente que reconstruir las mamas conlleva diversos riesgos para la salud y la calidad de vida de la mujer, pero en ciertos casos es necesario considerar su necesidad para salvaguardar su autoestima y estado emocional. A este respecto, Porroche (2013) plantea que la reconstrucción inmediata puede más bien dificultar la elaboración del duelo, pues es posible que evite la expresión de emociones como el temor, la ira o la tristeza, así como el proceso de redescubrir un nuevo cuerpo, aceptarlo y adaptarse a él.

En virtud de que una quinta parte de las pacientes sometidas a mastectomía optan por la re- 
construcción mamaria (Sepúlveda, 2016), es pertinente, en el campo de la psicología de la salud, revisar los avances de la literatura científica respecto al impacto que esta cirugía tiene en las personas que optan por ella. Por consiguiente, el objetivo principal de este trabajo fue identificar si la reconstrucción mamaria mejora la satisfacción con la imagen corporal y la calidad de vida de pacientes mastectomizadas. Tal tarea se llevó a cabo a través de una revisión sistemática y la aplicación de la prueba DIAD (por sus siglas en inglés) para el diseño e implementación de la metodología a la literatura hallada.

\section{MÉTODO}

\section{Universo}

Se realizó una búsqueda de la literatura científica publicada en inglés o en español, del año 2012 al mes de junio de 2017, referente a la recuperación de la satisfacción con la imagen corporal y la calidad de vida en mujeres con reconstrucción mamaria debida a la mastectomía por cáncer de mama.

A este efecto, se desarrolló un protocolo de trabajo denominado PICOS (Población-Intervención-Características del grupo control-Variables o constructos-Diseño de los estudios). A partir de la aplicación de este protocolo se lograron definir los criterios de búsqueda bibliográfica, llevar a cabo la de los artículos que cumplieran con los criterios establecidos, seleccionarlos según la prueba DIAD, elaborar el diagrama de flujo PRISMA, revisar de forma completa los textos seleccionados, analizar la información y presentar por último el informe correspondiente.

\section{Criterios de selección de artículos a partir de la aplicación del protocolo PICOS}

Se incluyeron aquellos artículos que implicaran mujeres mastectomizadas como población objeto, que dicha mastectomía fuera de tipo radical y simple, y que se hubiese originado como consecuencia de un cáncer de mama en mujeres de cualquier edad. No necesariamente debía haberse utilizado un grupo control en los estudios localizados, y estos podían ser descriptivos, correlacionales o cuasiex- perimentales. Los criterios de exclusión fueron a su vez los referidos a lumpectomías o mastectomías subcutáneas.

\section{Estrategias de búsqueda, selección y revisión de los estudios}

Para la realización de este estudio, la estrategia general de búsqueda fue la siguiente: (Mastectomía OR Reconstrucción mamaria) AND (Calidad de vida OR Imagen Corporal OR Satisfacción corporal); (Reconstrucción mamaria) AND (Calidad de vida).

(Reconstrucción mamaria) AND (Satisfacción corporal OR Imagen corporal).

Como fuentes documentales se tuvieron en cuenta bases de datos con sus respectivas rutas de búsqueda, como Science Direct: (reconstrucción mamaria OR cáncer de seno) AND (calidad de vida OR imagen corporal OR satisfacción corporal) (imagen corporal OR calidad de vida) AND (mastectomía OR cirugía reconstructiva) (reconstrucción mamaria OR mastectomía) AND (calidad de vida OR imagen corporal). Medline: (body image mastectomy); (reconstruction after mastectomy) AND (body image); (reconstruction after mastectomy) AND (body image and quality of life); $P u b$ Med: (body image breast cancer AND reconstruction after mastectomy); (body image breast cancer OR quality life AND reconstruction after mastectomy); (body perception AND reconstruction after mastectomy); (quality life AND reconstruction after mastectomy) (quality life AND reconstruction after mastectomy). Cochrane: (reconstrucción mamaria AND calidad de vida; calidad vida AND cáncer de mama; calidad vida AND reconstrucción mama; reconstrucción mama AND imagen corporal). Fueron particularmente útiles las fuentes clave en el tema, como Body Image y Revista Internacional de Psicología Clínica y de la Salud; algunos lugares virtuales específicos del área del conocimiento, como la División de Psicología de la Salud de la Asociación Americana de Psicología (APA), ciertos lugares en los que se encontró literatura "gris" o no publicada, como Google Scholar, y sitios web estratégicos como fuentes de información, como los de la OMS, la Organización Panamericana de la Salud (OPS) y los Institutos Nacionales de Salud (NIH). 
Para la selección de los artículos se aplicó la prueba DIAD (Valentine y Cooper, 2008), la cual permite evaluar la calidad de las intervenciones efectivas en investigación respecto al diseño e implementación de los tratamientos, teniéndose en cuenta en este caso tres factores: ajuste entre conceptos y operaciones, generalización de los hallazgos y precisión de la estimación de resultados. Una vez realizada la búsqueda, se aplicó el diagrama de flujo PRISMA (Figura I).

Figura 1. Diagrama de flujo PRISMA en el que se presenta el número de artículos identificados para el estudio.

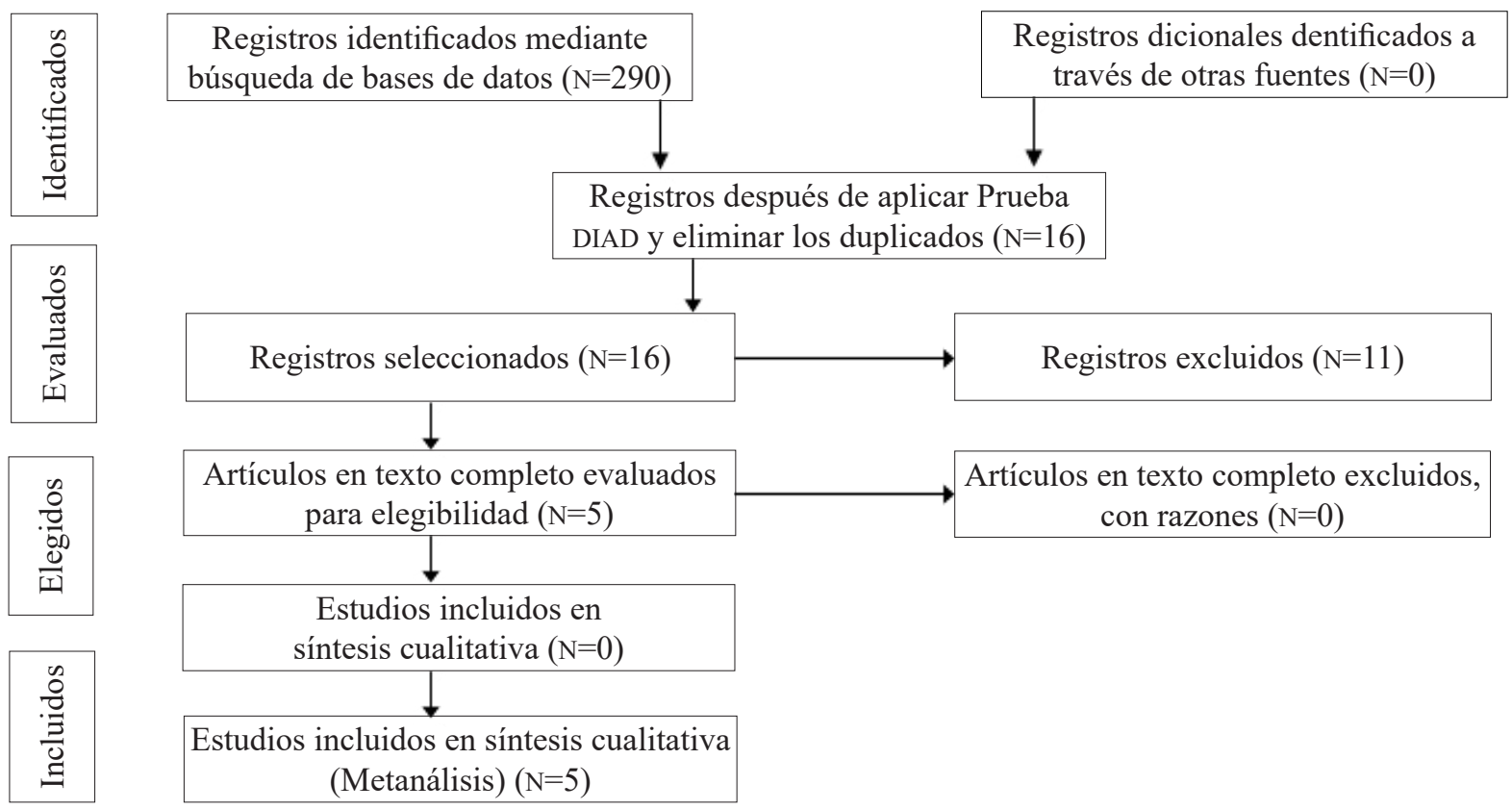

\section{RESULTADOS}

La Figura 1 ilustra que se identificaron 290 referencias en las bases de datos consultadas después de eliminar las duplicadas; al aplicar los criterios de la prueba DIAD, se seleccionaron 16 para la lectura completa, y de éstos se eligieron 5. Es impor- tante señalar que la eliminación de esos 11 artículos respondió al hecho de que no cumplían los indicadores de evaluación de calidad establecidos en la prueba DIAD, los cuales, por su rigor, no son alcanzables en dichas investigaciones. En la Tabla 1 se detallan los estudios incluidos.

Tabla 1. Resultados de la evaluación de los artículos por medio de la prueba DIAD.

\begin{tabular}{|c|c|c|c|}
\hline Referencia & $\begin{array}{l}\text { Factor 1: Ajuste } \\
\text { entre conceptos y operaciones }\end{array}$ & $\begin{array}{c}\text { Factor 2: Generalización } \\
\text { de los hallazgos }\end{array}$ & $\begin{array}{c}\text { Factor 3: Precisión } \\
\text { de la estimación de resultados }\end{array}$ \\
\hline $\begin{array}{l}\text { Figueroa, } \\
\text { Rivera } \\
\text { y Navarro } \\
(2016) .\end{array}$ & $\begin{array}{l}\text { La calificación obtenida a través } \\
\text { del cumplimiento de cada uno de } \\
\text { los criterios que aplicaban para } \\
\text { dicho factor fue } 0.57 / 1 \text {, lo que } \\
\text { se refleja en la correspondencia } \\
\text { entre la medición realizada de las } \\
\text { variables de satisfacción sexual } \\
\text { e imagen corporal en mujeres } \\
\text { mastectomizadas a raíz del cáncer } \\
\text { de mama. }\end{array}$ & $\begin{array}{l}\text { La calificación obtenida fue } \\
0.25 / 1 \text {, lo que indica que el nivel } \\
\text { de generalización de los resultados } \\
\text { es bajo; esto se evidencia en que si } \\
\text { bien el estudio identificó variables } \\
\text { relevantes, no se ajustó a la con- } \\
\text { dición de reconstrucción mamaría } \\
\text { luego de la mastectomía. }\end{array}$ & $\begin{array}{l}\text { Obtuvo una calificación de } 0.125 / 1 \\
\text { ya que carece de valores corres- } \\
\text { pondientes al tamaño del efecto. }\end{array}$ \\
\hline
\end{tabular}




\begin{tabular}{|c|c|c|c|}
\hline $\begin{array}{l}\text { Gómez, } \\
\text { Gómez, Lara } \\
\text { y Tejedor } \\
(2016) .\end{array}$ & $\begin{array}{l}\text { La puntuación alcanzada en este } \\
\text { caso fue de } 1 / 1 \text {, lo que indica total } \\
\text { cumplimiento. }\end{array}$ & $\begin{array}{l}\text { Este factor obtiene una calificación } \\
\text { de } 0.75 / 1 \text { en razón de que se contó } \\
\text { con participantes que cumplían las } \\
\text { características requeridas, se uti- } \\
\text { lizaron instrumentos de medición } \\
\text { pertinentes, y se logró identificar } \\
\text { otro tipo de variables que permiten } \\
\text { comprender mejor el cambio en la } \\
\text { satisfacción con la imagen corpo- } \\
\text { ral y la calidad de vida. }\end{array}$ & $\begin{array}{l}\text { La calificación fue } 0 / 1 \text { ya que no } \\
\text { se muestran indicadores estadís- } \\
\text { ticos que permitan contrastar y } \\
\text { evidenciar los resultados enuncia- } \\
\text { dos por los autores. }\end{array}$ \\
\hline $\begin{array}{l}\text { Brennan, } \\
\text { Flitcroft, } \\
\text { Warrier, } \\
\text { Snook } \\
\text { y Spillane } \\
(2016) .\end{array}$ & $\begin{array}{l}\text { Se alcanzó un resultado de } 0.57 / 1 \text {, } \\
\text { pues se logran medir y describir } \\
\text { las variables de satisfacción con } \\
\text { la imagen corporal y la calidad de } \\
\text { vida en mujeres con reconstruc- } \\
\text { ción mamaria. }\end{array}$ & $\begin{array}{l}\text { Hay una valoración de } 0.75 / 1 \text {, por } \\
\text { lo que se puede decir que hay un } \\
\text { cumplimiento medianamente alto. }\end{array}$ & $\begin{array}{l}\text { La estimación fue } 0.38 / 1 \text { dado } \\
\text { que no se muestran indicadores } \\
\text { estadísticos que hagan posible } \\
\text { contrastar y evidenciar los resul- } \\
\text { tados enunciados por los autores. } \\
\text { Sin embargo, se exponen esta- } \\
\text { dísticos de las subescalas de los } \\
\text { instrumentos utilizados para medir } \\
\text { las variables. }\end{array}$ \\
\hline $\begin{array}{l}\text { Rincón, Pérez, } \\
\text { Borda } \\
\text { y Martín } \\
(2012) .\end{array}$ & $\begin{array}{l}\text { Con una calificación de } 0.86 / 1 \text {, } \\
\text { el artículo permite identificar el } \\
\text { impacto de la reconstrucción ma- } \\
\text { maria en la imagen corporal y la } \\
\text { autoestima, pero no en la calidad } \\
\text { de vida. }\end{array}$ & $\begin{array}{l}\text { Se logra un puntaje de } 0.88 / 1 \text {, lo } \\
\text { que indica que la muestra cumple } \\
\text { con las características deseadas. }\end{array}$ & $\begin{array}{l}\text { La valoración fue } 0.25 / 1 \text { ya que no } \\
\text { se muestran indicadores estadís- } \\
\text { ticos que permitan contrastar y } \\
\text { evidenciar los resultados enuncia- } \\
\text { dos por los autores. }\end{array}$ \\
\hline $\begin{array}{l}\text { El Troudi et } \\
\text { al. }(2017) \text {. }\end{array}$ & $\begin{array}{l}\text { En este factor se obtiene una califi- } \\
\text { cación de } 0.29 / 1 \text {, lo que indica un } \\
\text { bajo cumplimiento de ajuste entre } \\
\text { los conceptos y su medición. }\end{array}$ & $\begin{array}{l}\text { Se obtiene } 0.50 / 1 \text {, ya que si bien } \\
\text { las características de la población } \\
\text { cumplen con los requisitos, las } \\
\text { pacientes refieren subjetivamente } \\
\text { una mejoría en su imagen corporal } \\
\text { y calidad de vida. }\end{array}$ & $\begin{array}{l}\text { La calificación fue } 0.25 / 1 \text { ya que } \\
\text { no se muestran indicadores esta- } \\
\text { dísticos que posibiliten contrastar } \\
\text { y evidenciar los resultados enun- } \\
\text { ciados por los autores. }\end{array}$ \\
\hline
\end{tabular}

Nota: La tabla muestra las calificaciones obtenidas en cada trabajo en los tres factores evaluados constitutivos de la prueba DIAD. Los puntajes podían oscilar entre 0 y 1 , siendo 1 la puntuación máxima en cada factor.

Como resultado de la revisión sistemática y aplicación de la prueba DIAD, se describen a continuación los artículos elegidos con el fin de comprender la puntuación obtenida y señalar algunos aspectos pertinentes en cuanto al propósito de la búsqueda misma y al tema de interés.

Como primer artículo se encuentra el elaborado por Figueroa, Rivera y Navarro (2016), quienes realizaron un estudio descriptivo y transversal en una muestra no probabilística por conveniencia de 120 mujeres diagnosticadas con algún tipo de cáncer de mama; por ende, no hubo grupos comparativos ni especificación alguna acerca del tipo de mastectomía o de reconstrucción mamaria. El instrumento utilizado para evaluar la calidad de vida fue el módulo QLQ-BR23 del EORT-C30.

Se halló que las mujeres con cáncer de mama consideran la sexualidad como un área irrelevante en su vida, sobre todo cuando la contrastan con la magnitud del tratamiento de una enfermedad cuyos efectos secundarios minan su salud e inte- gridad psíquica. No olvidan que la sexualidad no es solamente una relación coital, sino que implica también cercanía y proximidad física y emocional, lo que las apoya para reasegurar su vínculo de pareja. Sin embargo, la relación conyugal experimenta más estrés, ansiedad y preocupación, lo que se suma a la lucha de estas mujeres por sobrevivir al cáncer. Ellas, al haberse alejado sustancialmente del ejercicio de la sexualidad, evitan la reflexión sobre las implicaciones en su calidad de vida. Los autores reportan que para estas mujeres las relaciones sexuales son solo parte de una obligación derivada de su relación marital, actitud que puede estar relacionada con una sintomatología depresiva no diagnosticada en el mismo proceso de tratamiento, o bien con los duelos derivados de la pérdida de la salud.

Gómez, Gómez, Lara y Tejedor (2016) 1levaron a cabo un estudio retrospectivo a lo largo de siete años en el que participaron 31 mujeres a las que se les hizo una mastectomía y recons- 
trucción inmediata debido a un cáncer de mama no metastático, con axila clínicamente negativa. A ese efecto, conformaron dos grupos: el primero con 17 pacientes con reconstrucción con expansor-prótesis, excluyéndose aquellas que aún tuvieran el expansor, y un segundo grupo con 14 pacientes con colgajo muscular de dorsal ancho y prótesis. El cuestionario utilizado fue el MBROS Body Image Questionnaire.

Los autores encuentran que la imagen corporal parece haberse visto más afectada en el grupo de pacientes con mastectomía y expansor-prótesis, lo que puede deberse a que este grupo de mujeres necesitó la colocación de un expansor durante un tiempo y una cirugía posterior, lo que puede explicar la mayor alteración de la imagen corporal. Las secuelas psicológicas sobre dicha imagen y la sexualidad fueron mayores en este grupo de pacientes cuando se les comparó con pacientes mastectomizadas a las que se les realizó una reconstrucción con colgajo muscular de dorsal ancho y prótesis.

A su vez, Brennan, Flitcroft, Warrier, Snook y Spillane (2016) condujeron un estudio de tipo transversal en el que participaron 47 mujeres diagnosticadas con cáncer de mama y sometidas a radioterapia, a quienes la reconstrucción mamaria se les hizo mediante implantes y expansores. Los instrumentos que se utilizaron en esta investigación fueron el FACT-B para medir la calidad de vida, el BREAST-Q para evaluar la satisfacción, la Escala Body Image para la imagen corporal, y las escalas de Impacto de los Eventos y de Arrepentimiento Decisional el estrés.

Este grupo de trabajo pudo demostrar los buenos resultados cosméticos y de calidad de vida para las pacientes sometidas a la reconstrucción mamaria, una tasa de complicaciones importantes aceptablemente baja, y a la vez un arrepentimiento reducido por la decisión tomada. Cuando hubo una indicación temprana de que el resultado final podría ser subóptimo, el expansor tisular fue reemplazado por una reconstrucción autóloga, lo que tuvo beneficios en la reconstrucción autóloga retrasada. Las mujeres con tumores de alto riesgo valoraban la oportunidad de someterse a una reconstrucción mamaria y estaban dispuestas a aceptar las limitaciones de la intervención. Así, cuando se hace una elección informada, se pueden lograr altos niveles de satisfacción.
Rincón, Pérez, Borda y Martín (2012) constituyeron dos grupos para llevar a cabo su estudio: uno formado por mujeres que habían sido intervenidas mediante mastectomía sin reconstrucción mamaria posterior (Grupo 1) y otro con cirugía reconstructiva (Grupo 2). Estos dos grupos se subdividieron a su vez en cuatro subgrupos, cada uno integrado por dieciocho pacientes: pacientes con mastectomía simple unilateral sin reconstrucción mamaria; pacientes con mastectomía radical modificada unilateral sin reconstrucción mamaria; pacientes con reconstrucción mamaria inmediata, y pacientes con reconstrucción mamaria diferida. Los instrumentos que se utilizaron fueron una encuesta psicosocial, la Escala de Autoestima de Rosenberg, el Cuestionario de Calidad de Vida para Cáncer de Mama EORTC QLQ-BR 23 y el Test de Medición del Grado de Satisfacción Corporal. Los autores plantean que el tiempo transcurrido desde el diagnóstico oncológico y la intervención quirúrgica es una variable relevante en la calidad de vida de estas pacientes, cuya autoestima tiene relación con el nivel de satisfacción con el resultado quirúrgico y el deterioro de la imagen corporal. En general, hubo una mejoría en la satisfacción corporal y la autoestima, pero no en el funcionamiento sexual ya que una cierta insatisfacción permanece respecto a la zona del cuerpo de pecho/tórax, y también influye el tipo de mastectomía previa; por ejemplo, la radical modificada, que implica un vaciamiento axilar completo y extirpación de toda la glándula mamaria, trae consigo mayores molestias físicas que afectan la funcionalidad del cuerpo.

El Troudi et al. (2017), en un estudio prospectivo, observacional, descriptivo y comparativo a manera de ensayo clínico, analizaron a 36 pacientes que, a raíz de un cáncer de mama, accedieron a la reconstrucción mamaria, 20 de ellas con colgajo miocutáneo TRAM y 16 con colgajo dorsal ampliado. En la evaluación postoperatoria subjetiva no se encuentra definido el instrumento utilizado, aunque para identificar la depresión y ansiedad los autores utilizaron la escala HAD. Logran establecer que, respecto a su satisfacción con la imagen corporal, 55\% de las pacientes refirieron estar "muy satisfechas" con la reconstrucción TRAM; en el grupo dorsal ampliado, 94\% se mostraron igualmente muy satisfechas. Es importante 
señalar que las pacientes medianamente satisfechas y las no satisfechas presentaron complicaciones posoperatorias. Los investigadores también aplicaron la escala HAD a las pacientes en el postoperatorio, mediante la cual la mayoría $(75 \%$ en el grupo TRAM y $87.5 \%$ en el grupo dorsal ampliado) fueron catalogadas en un nivel normal; es decir, no se mostraron ansiosas ni depresiva, aceptaron satisfactoriamente su nueva apariencia física, y solo una paciente del grupo TRAM, que tuvo dos complicaciones, se catalogó como grave dada la puntuación que obtuvo al realizar el test.

\section{DISCUSIÓN}

La presente revisión sistemática, hecha con la prueba DIAD, arrojó un número de artículos limitado a pesar de los 290 encontrados inicialmente sin ningún tipo de filtro de calidad. Al aplicárseles los criterios de este protocolo, propios para estudios de tratamientos clínicos, dichos artículos se redujeron solamente a cinco, lo que pone en evidencia el hecho de que las investigaciones clínicas en el campo de la salud no siempre se diseñan con indicadores relativos a la coherencia entre la intervención y el efecto esperado, entre el diseño propuesto para determinar una relación causal entre la intervención y su efectividad, y entre la presentación de estimaciones precisas del impacto de la intervención y la capacidad del estudio para generalizar los hallazgos. Este resultado coincide con el obtenido por Guyomard, Leinster y Wilkinson (2007) en su revisión sistemática, misma que resalta las deficiencias metodológicas en la investigación publicada para evaluar la satisfacción con la reconstrucción mamaria basada en la evidencia.

Mediante la revisión hecha, es posible plantear, tal como lo expresa Ortiz (2016), que el interés sexual de la mujer puede verse mermado tras el diagnóstico de cáncer de mama y la mastectomía subsecuente en razón de los sentimientos de baja autoestima, pobre autoimagen, pérdida de identidad femenina, disminución del atractivo físico e incluso disfunción sexual en algunas mujeres, lo que repercute en su actividad sexual y acarrea serias repercusiones a la relación de pareja.

En general, la mastectomía se asocia con mayores niveles de depresión, imagen corporal más negativa, baja autoestima y pobre funcionamiento sexual (Cucarella, 2013; Fernández, 2004; Sánchez, 2015; Segura et al., 2014), lo que no mejora sustancialmente con la cirugía reconstructiva (Figueroa et al., 2016; Frech, 2016), aun cuando autores como Sinaei et al. (2017) señalan que estas mujeres mejoran su satisfacción con la imagen corporal y calidad de vida.

Las mastectomías realizadas con las técnicas de colgajos muestran mejores resultados sobre estos aspectos que aquellas que emplean expansores. Ante tal resultado, autores como Edsander-Nord, Brandberg y Wickman (2001), Veiga et al. (2004), Cabrera et al. (2006), Tønseth, Hokland, Tindholdt, Åbyholm y Stavem (2008), Yueh et al. (2010) y Santosa et al. (2018) expresan que la reconstrucción mamaria con colgajo ha sido una técnica eficaz que logra un elevado porcentaje de satisfacción y mejoramiento de la imagen corporal, incluso superior al logrado en estudios previos en los que se ha valorado la satisfacción con la reconstrucción mamaria y la imagen corporal mediante expansores. En este sentido, es importante reconocer que si bien la reconstrucción mamaría es una cirugía que mejora la vida y a la satisfacción con la imagen corporal de las mujeres mastectomizadas, hay tipos de cirugías, como las realizadas con colgajos, que tienen resultados más positivos en esos aspectos.

El papel y actitud de la paciente ante al diagnóstico, el tratamiento oncológico y la reconstrucción mamaria determinan en cierta medida sus niveles de satisfacción personal, tal como lo expresan Brennan et al. (2016). Acerca de este aspecto, Deutsch (2016) recomienda que el equipo médico se asegure de que la paciente ingresa a cirugía con la mayor información posible, incluidos el riesgo que representa, sus posibles complicaciones y los beneficios que entraña, asumiendo que este tipo de intervención implica una elevada carga emocional para la mujer.

En los estudios clínicos es necesario establecer metodologías rigurosas en cuanto a la determinación de muestras, elección de instrumentos, tratamientos, causalidad y procedimientos estadísticos que permitan cuantificar los efectos del tratamiento y la presentación de resultados, a fin de encontrar respuestas confiables y avanzar así en la construcción del conocimiento. 
Hay un acuerdo general de que la reconstrucción mamaria mejora las condiciones y la calidad de vida en mujeres mastectomizadas; sin embargo, toda vez que hay tipos de cirugías como la que utiliza colgajos o las autólogas, que ayudan aún más a la recuperación de la satisfacción con la imagen corporal, es importante promover intervenciones que impacten de manera positiva la vida de las personas.

A pesar de la reconstrucción mamaria, el ejercicio de la sexualidad femenina sigue viéndose afectado negativamente debido al significado de las mamas en la idea de ser mujer y de su rol como ser sexuado y activo dentro de una relación de pareja, que se inhibe mucho más cuando se daña debido a la enfermedad. Sobre todo, es un reto para los equipos de apoyo psicosocial de las unidades oncológicas.

Los estudios revisados permiten ver que es importante incluir a la paciente - a partir de una comunicación abierta y una información suficiente- en el proceso de intervención quirúrgica. Este hecho le permitirá tomar una decisión más consciente y tener una participación más activa, lo que favorecerá la satisfacción con el proceso y con su autoimagen, repercutiendo positivamente en su calidad de vida.

Por último, a pesar de los beneficios que trae consigo la reconstrucción mamaria, es inevitable el efecto negativo de la mastectomía sobre la imagen corporal, por lo que es necesario seguir trabajando desde la psicología de la salud para aminorar o evitar ese efecto.

\section{REFERENCIAS}

Agencia Internacional de Investigación en Cáncer (2012). El cáncer de mama en las Américas. Recuperado de http://globocan. iarc.fr/. The World Bank Group 2014 Indicators.

Álvarez H., C., Brusint, B., Vich, P., Díaz G., N., Cuadrado R., C. y Hernández G., M. (2015). Actualización del cáncer de mama en atención primaria. Semergen-Medicina de Familia, 41(1), 34-47. doi: 10.1016/j.semerg.2014.03.004.

Bindu, N.P.y Baboo, G. (2017). Effect of cosmetic surgery on body image and body image specific quality of life. Journal of the Indian Academy of Applied Psychology, 43(1), 157.

Breastcancer.org (2016). Tipos de reconstrucción mamaria. Recuperado de http://www.breastcancer.org/es/tratamiento/cirugia/ reconstruccion/tipos.

Brennan, M.E., Flitcroft, K., Warrier, S., Snook, K. y Spillane, A.J. (2016). Immediate expander/implant breast reconstruction followed by post-mastectomy radiotherapy for breast cancer: Aesthetic, surgical, satisfaction and quality of life outcomes in women with high-risk breast cancer. The Breast, 30, 59-65. Recuperado de https://www.sciencedirect.com/science/article/ pii/S0960977616301448.

Bruant-Rodier, C. y Bodin, F. (2014). Reconstrucción mamaria. Cirugía Plástica Reparadora y Estética, 22(1), 1-25. doi: 10.1016/ S1634-2143(14)67578-6.

Cabrera, E., Redondo, A., Dean, A., Benítez, J.R., Torre, C., De Piero, G., Navarro, J., Molina, H., Ramírez, J.N. y Rioja, L.F. (2006). Satisfacción en pacientes con reconstrucción mamaria con colgajo D.I.E.P. Cirugía Plástica Ibero-Latinoamericana, 32(3), 169-179. Recuperado de http://scielo.isciii.es/scielo.php?script=sci_arttextypid=S0376-78922006000300003ylng=esytlng=pt.

Cruz B., L. y Morales H., E. (2014). Historia y estado actual sobre los tipos de procedimientos quirúrgicos realizados en cáncer de mama. Gaceta Mexicana de Oncología, 13(2), 124-133.

Cucarella, M. (2013). Bienestar emocional, imagen corporal, autoestima y sexualidad en mujeres con cáncer de mama (Disertación Doctoral). Valencia (España): Universitat de València, Recuperado de http://roderic.uv.es/handle/10550/26371.

Deutsch, C. J. (2016). Patients having breast reconstruction should enter into surgery with as much information as possible. British Medical Journal, 352, i1712. Recuperado de http://www.bmj.com/content/352/bmj.i1712.full.

Edsander-Nord, A., Brandberg, Y. y Wickman, M. (2001). Quality of life, patients' satisfaction, and aesthetic outcome after pedicled or free TRAM flap breast surgery. Plastic and Reconstructive Surgery, 107(5), 1142-1153.

El Troudi M., A.T., Duque L., V., Ortiz J., D.D., Angulo, B., Portilla, J. y Daza D., J.R. (2017). Mastectomía radical con reconstrucción inmediata en el Instituto Autónomo Hospital Universitario de Los Andes, período junio 2012-abril 2015. Revista Chilena de Cirugía, 69(3), 234-246. doi: 10.1016/j.rchic.2016.11.012.

Fernández A., I. (2004). Alteraciones psicológicas asociadas a los cambios en la apariencia física en pacientes oncológicos. Psicooncología, 1(2), 169-180. 
Figueroa V., R.M., Rivera H., M.E. y Navarro H., M.R. (2016). «No importa»: sexualidad femenina y cáncer de mama. Gaceta Mexicana de Oncología, 15(5), 278-284. doi 10.1016/j.gamo.2016.09.003.

Frech, G. (2016). Calidad de vida en mujeres con cáncer de mama sometidas a mastectomía más reconstrucción mamaria y mastectomía sin reconstrucción que fueron atendidas en el Hospital Militar "Alejandro Dávila Bolaños en el periodo enero 2012 a enero 2015. (Disertación doctoral). Managua: Universidad Nacional Autónoma de Nicaragua. Recuperado de http:// repositorio.unan.edu.ni/3043/.

Gallegos, M., Pérez, C. y Bustamante, S. (2017). El cuidado de enfermería a la mujer; cuerpo, cáncer, mastectomía y sus significados. Bloomington, IN: Palibrio.

Gómez, T., Gómez, S., Lara, C. y Tejedor, L. (2016). Satisfacción de la reconstrucción mamaria tras mastectomía: diferencias entre la colocación de expansor-prótesis y uso del colgajo de dorsal ancho. Revista de Senología y Patología Mamaria, 29(1), 19-25. doi: 10.1016/j.senol.2015.11.001.

Guyomard, V., Leinster, S. y Wilkinson, M. (2007). Systematic review of studies of patients' satisfaction with breast reconstruction after mastectomy. The Breast, 16(6), 547-567.

Instituto Nacional del Cáncer (2016). Reconstrucción del seno después de una mastectomía. Recuperado de https://www.cancer. gov/espanol/tipos/seno/hoja-informativa-reconstruccion.

Marré, D., Gantz, J.T., Villalón, J. y Roco, H. (2016). Reconstrucción mamaria: estado actual del tema. Revista Chilena de Cirugía, 68(2), 186-193. doi: 10.4067/S0718-40262016000200014.

Martínez B., A.E., Lozano A., A., Rodríguez V., A.L., Galindo V., Ó. y Alvarado A., S. (2014). Impacto psicológico del cáncer de mama y la mastectomía. Gaceta Mexicana de Oncología, 13(1), 53-58.

Mejía R., M.E. (2012). Incertidumbre, calidad de vida e imagen corporal en mujeres sometidas a mastectomía (Tesis de Doctora en Enfermería). Bogotá: Universidad Nacional de Colombia. Recuperado de: http://www. bdigital. unal. edu. co/11530/1/ mariaelenamejiarojas.

Morales C., M.S., Torres B., M. y Vega M., A.M.. (2016). La mastectomía: una mirada social desde la fisioterapia. Revista de la Facultad de Medicina, 64(Supl. 1), 53-58.

Negenborn, V.L., Young-Afat, D.A., Dikmans, R.E.G., Smit, J.M., Winters, H.A.H., Griot, J.P. W.D. y Moerman, E. (2018). Quality of life and patient satisfaction after one-stage implant-based breast reconstruction with an acellular dermal matrix versus two-stage breast reconstruction (BRIOS): primary outcome of a randomised, controlled trial. The Lancet Oncology, 19(9), 1205-1214.

Organización Mundial de la Salud (2017). Cáncer: Datos y cifras. Ginebra: OMS. Recuperado de http://www.who.int/mediacentre/factsheets/fs297/es/.

Ortiz R., C. (2016). La vivencia de la sexualidad en la mujer mastectomizada (Trabajo de Fin de Grado). Madrid: Universidad Autónoma de Madrid. Recuperado de: https://repositorio.uam.es/bitstream/handle/10486/675746/ortiz_rozalen_cristinatfg.pdf?sequence=1.

Porroche, A. (2013). Luces y sombras de la reconstrucción mamaria. Mujer y Salud, 34, 30-33. Recuperado de http://sro.sussex. ac.uk/47584/1/Porroche_MyS_Luces_y_Sombras.pdf.

Rincón M., E., Pérez M., Á., Borda, M. y Martín, A. (2012). Impacto de la reconstrucción mamaria sobre la autoestima y la imagen corporal en pacientes con cáncer de mama. Universitas Psychologica, 11(1), 25-41.

Román, J.M. (2007). La cirugía en el cáncer de mama. Psicooncología, 4(2), 301-310.

Rosenberg, M. (1965). Society and the adolescent self-image. Princeton, NJ: Princeton University Press.

Sánchez S., T. (2015). Efectos psíquicos de la mastectomía (preventiva y terapéutica). Cambios en la percepción de las mujeres con cáncer de mama. Papeles del Psicólogo, 36(1), 62-73.

Santosa, K.B., Qi, J., Kim, H.M., Hamill, J.B., Wilkins, E.G. y Pusic, A.L. (2018). Long-term patient-reported outcomes in postmastectomy breast reconstruction. Journal of the American Medical Association Surgery, 153(10), 891-899. doi: 10.1001/ jamasurg.2018.1677.

Segura V., M., García N., R. y Gutiérrez, L. (2014). Imagen corporal y autoestima en mujeres mastectomizadas. Psicooncología, 11(1), 45-57. doi: 10.5209/rev_PSIC.2014.v11.n1.44916.

Sepúlveda, S. (2016). Reconstrucción mamaria. Revista Médica de la Clínica Las Condes, 27(1), 65-75. Recuperado de https:// www.sciencedirect.com/science/article/pii/S0716864016000109.

Sinaei, F., Zendehdel, K., Adili, M., Ardestani, A., Montazeri, A. y Mohagheghi, M.A. (2017). Association between breast reconstruction surgery and quality of life in Iranian breast cancer patients. Acta Medica Iranica, 55(1), 35-41. Recuperado de http://acta.tums.ac.ir/index.php/acta/article/view/5121.

Tønseth, K.A., Hokland, B.M., Tindholdt, T.T., Åbyholm, F.E. y Stavem, K. (2008). Quality of life, patient satisfaction and cosmetic outcome after breast reconstruction using DIEP flap or expandable breast implant. Journal of Plastic, Reconstructive \& Aesthetic Surgery, 61(10), 1188-1194.

Tumas, N., Díaz M., D.P. y Carbonetti A., C. (2013). Feminismo y cáncer de mama, una estrecha relación. Memorias de las III Jornadas CINIG de Estudios de Género y Feminismos, 25-27 de septiembre, La Plata, Argentina. Recuperado de http:// sedici.unlp.edu.ar/handle/10915/41888. 
Valentine, J.C. y Cooper, H. (2008). A systematic and transparent approach for assessing the methodological quality of intervention effectiveness research: the Study Design and Implementation Assessment Device (Study DIAD). Psychological Methods, 13(2), 130-149. Recuperado de http://psycnet.apa.org/fulltext/2008-06808-004.html.

Veiga, D.F., Neto, M.S., Ferreira, L.M., Garcia, E.B., Veiga F., J., Novo, N.F. y Rocha J., L.B.S. (2004). Quality of life outcomes after pedicled TRAM flap delayed breast reconstruction. British Journal of Plastic Surgery, 57(3), 252-257.

Villamil M., M.L., Choachí, J., Gómez Á., P., León, L., Ovalle Á., M., Rodas L., F... y Soto N., A. (2012). Mujeres con cáncer de seno: experiencias y significados. Revista Colombiana de Enfermería, 7(7), 12-21. Recuperado de https://dialnet.unirioja. es/servlet/articulo? codigo $=4066200$.

Yueh, J.H., Slavin, S.A., Adesiyun, T., Nyame, T.T., Gautam, S., Morris, D.J. y Lee, B.T. (2010). Patient satisfaction in postmastectomy breast reconstruction: a comparative evaluation of DIEP, TRAM, latissimus flap, and implant techniques. Plastic and Reconstructive Surgery, 125(6), 1585-1595.

Yustos M., A., Angulo M., M. y Soto M., Á.M. (2017). Cáncer de mama. Medicine. Programa de Formación Médica Continuada Acreditado, 12(34), 2011-2023. doi: 10.1016/j.med.2017.05.001. 